\title{
Measuring Segmented Primary Mirror WFE in the Presence of Vibration and Thermal Drift on the Light-Weighted JWST
}

Tony L. Whitman, Kenneth J. Dziak, Conrad Wells, Gene Olczak

ITT Exelis Geospatial Systems, 400 Initiative Drive, P.O. Box 60488, Rochester, NY, USA 14606

\begin{abstract}
The light-weighted design of the Optical Telescope Element (OTE) of the James Webb Telescope (JWST) leads to additional sensitivity to vibration from the ground - an important consideration to the measurement uncertainty of the wavefront error (WFE) in the primary mirror. Furthermore, segmentation of the primary mirror leads to rigid-body movements of segment areas in the WFE. The ground vibrations are minimized with modifications to the test facility, and by the architecture of the equipment supporting the load. Additional special test equipment (including strategically placed isolators, tunable mass dampers, and cryogenic magnetic dampers) mitigates the vibration and the response sensitivity before reaching the telescope. A multi-wavelength interferometer is designed and operated to accommodate the predicted residual vibration. Thermal drift also adds to the measurement variation. Test results of test equipment components, measurement theory, and finite element analysis combine to predict the test uncertainty in the future measurement of the primary mirror. The vibration input to the finite element model comes from accelerometer measurements of the facility with the environmental control pumps operating. One of the isolators have been built and tested to validate the dynamic performance. A preliminary model of the load support equipment and the OTE with the Integrated Science Instrument Module (ISIM) is complete. The performance of the add-on dampers have been established in previous applications. And operation of the multi-wavelength interferometer was demonstrated on a scaled hardware version of the JWST in an environment with vibration and thermal drift.
\end{abstract}

Keywords: James Webb Space Telescope, interferometry, large segmented mirror, vibration

\section{INTRODUCTION}

The JWST primary mirror surface figure will be measured as an aggregate for the first time after integration of the OTE and ISIM by a multi-wavelength interferometer located at the center of curvature of the mirror. Vibration contributes uncertainty in the figure measurement with movement between mirror segments and variation in the alignment between the mirror and the interferometer. Temporal variations in the relative positions of the mirror segments are manifested as primary mirror figure error. The variation in the alignment of the mirror relative to the interferometer adds fringe density contributing to measurement noise and, potentially, aliasing. The multiple-wavelength interferometer can align and phase the mirror segments with step heights between segments of more than one millimeter by combining phase maps of differing wavelengths to remove phase ambiguity. Movement of the mirrors between phase maps also adds uncertainty in the phasing of the segments.

The source of the vibration is the ground and facility. Chamber $A$ at the Johnson Space Center is one of the few cryogenic vacuum chambers large enough for the JWST optical test. The chamber was designed and built for testing of the hardware and personnel of the Apollo mission in the 1960's rather than optimized for a 21 st century interferometer test - complete with operating vacuum pumps.

To minimize the effects due to vibration, the mechanical support couples the interferometer to the telescope and isolates the vibration at the chamber interface. One of the isolators has been built and tested. Details of the design and test are below. A finite-element model (FEM) generates predictions of the vibration effects and guides the strategic placement of mass and magnets to dampen the sensitivity to vibration. Then further error reduction results with the use of data averaging to meet the needs of the optical test, and has been demonstrated on the JWST $1 / 6$ th-scale testbed telescope in a laboratory. 


\section{TEST CONFIGURATION DESIGN}

Figure 1 illustrates the design representation of the test configuration. The JWST rests on a cradle called the Hardpoints and Offloaders Support Subsystem (HOSS). The HOSS suspends from 6 Telescope Rods in a hexapod configuration shown more visibly in Figure 2. The rods in hexapod form couple the HOSS rigidly to the Upper Support Frame (USF), a truss supporting auto-collimating flat (ACF) mirrors and the Center of Curvature Optical Assembly with the interferometer. Therefore, the primary mirror and the interferometer are rigidly coupled and move together to maintain optical alignment nearly independent of chamber movement. The USF hangs by 6 Down Rods from vibration isolators designed by Minus K Technology.

Underneath the HOSS are 8 magnetic dampers in 4 orthogonal pairs between the HOSS and the chamber floor to mitigate the pendulum motion of the suspended system as shown in Figure 3. These are permanent magnets at cryogenic temperatures using a strong magnetic field to dampen the low frequency pendulum motion without transmitting the floor vibration to the HOSS. 


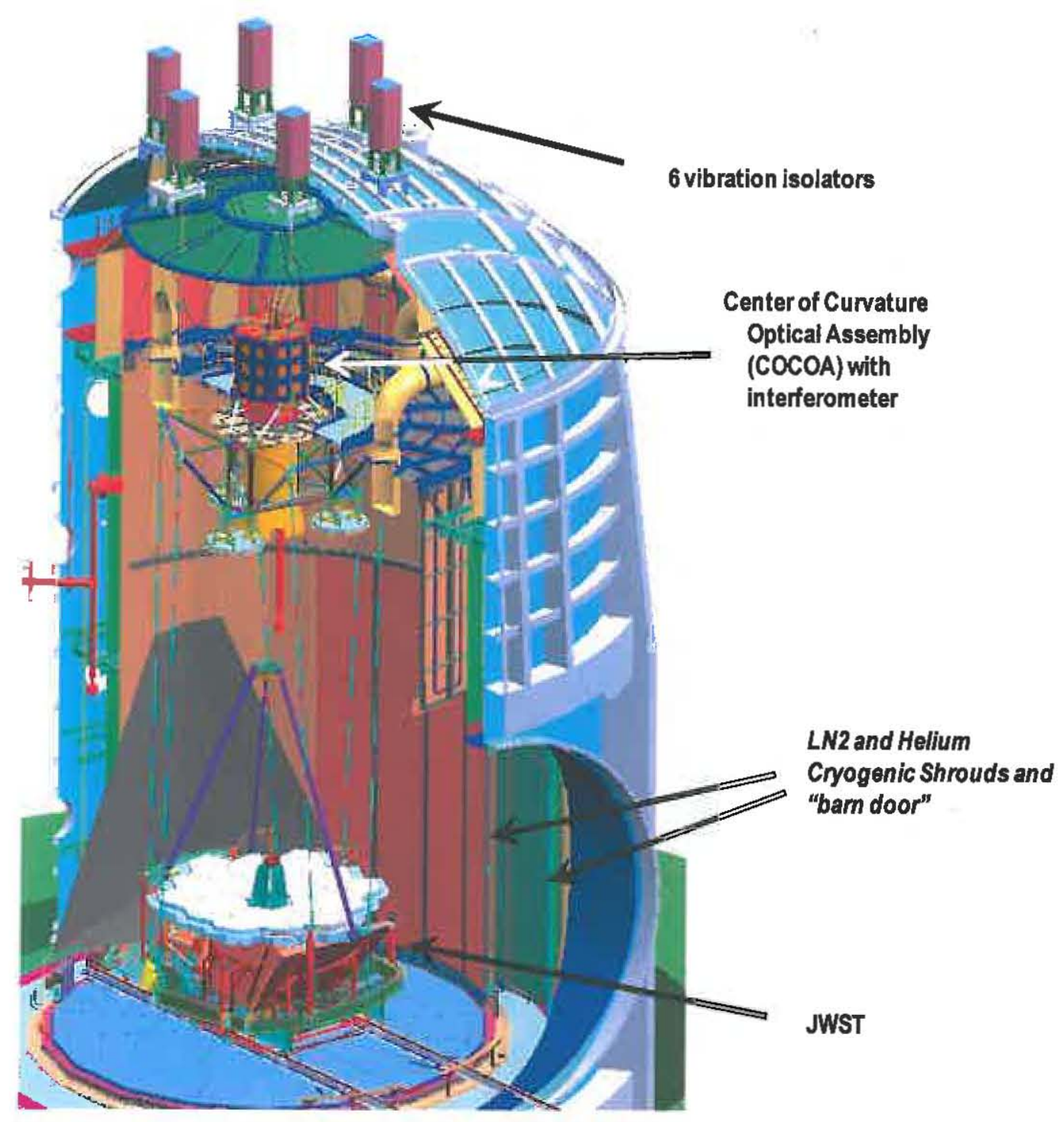

Figure 1 Optical Test Configuration of JWST 


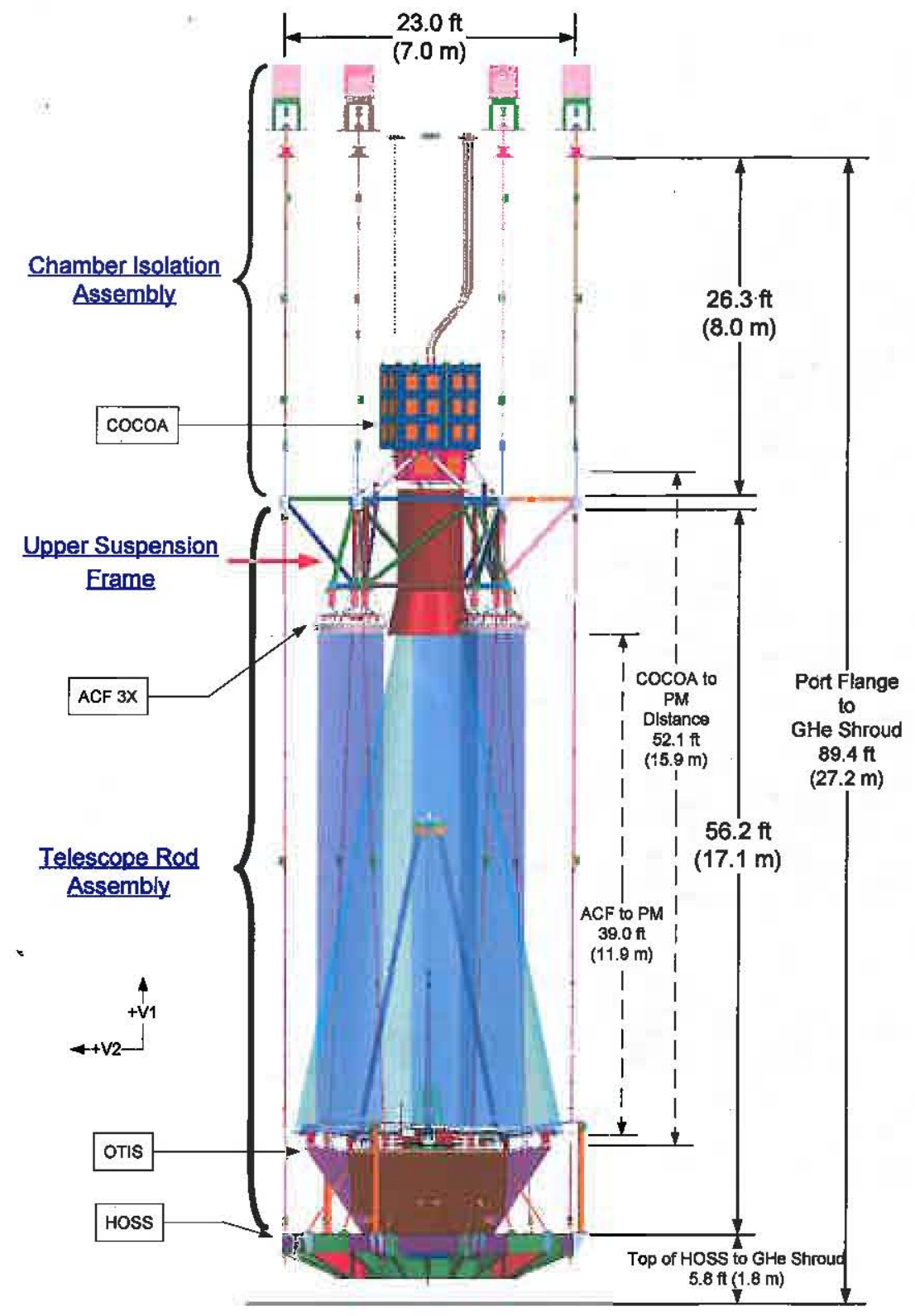

Figure 2 Side view of suspended JWST

\section{VIBRATION SOURCE AND MITIGATION}

Chamber A was tested while operating the cryogenic vacuum equipment in 2008 and 2009 using accelerometers (by Wilcoxon and others) in three orthogonal orientations at multiple locations on the chamber. An example power spectral density (PSD) in one direction is shown in Figure 4. The facility is currently in renovation with updated quieter pumps with mount isolation and the addition of helium refrigeration equipment. The renovation likely shifts the PSD peak locations in frequency, so the input to the FEM envelope the magnitude of the PSD with allowances for shifts in frequency. The accelerometers will measure the facility again after completion of the renovations in 2012. 


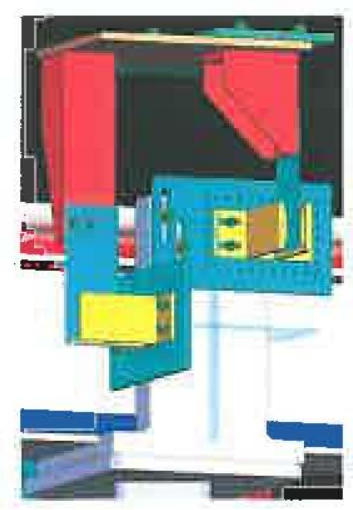

Figure 3 Schematic of a magnetic damper pair between the HOSS and the chamber floor.

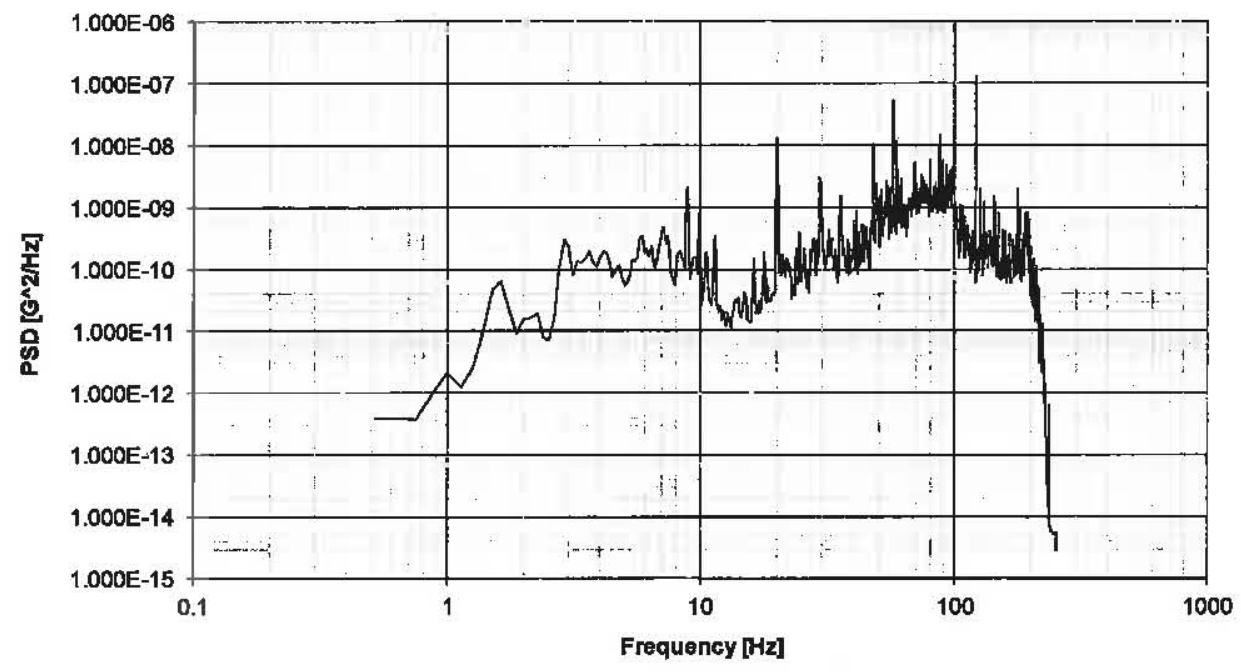

Figure 4 Vibration measured at JSC Chamber A before renovation for JWST test

Isolators attenuate transmission of the facility vibration above $7 \mathrm{~Hz}$. Figure 5 shows one isolator in test with a stinger and shaker to generate a graph of the transmissibility of the isolator and to validate the FEM model representation of the isolator.

After building an FEM of the suspension system and combining the FEM of the isolator and JWST, predictions of the mirror segments relative to each other and relative to the interferometer led to additional vibration dampers in strategic locations for further reduction in the optical measurement. The primary mirror has two wings of mirror segments that fold back for launch. The potential for movement of the wings relative to the center section during the test drives the need for dampers. Likewise, dampers are placed on each of the 3 struts holding the secondary mirror to dampen potential movement require a damper. Underneath several components of JWST are several gravity offloaders supported by the HOSS that need dampers. The Upper Support Frame between the telescope rods and the COCOA have 3 dampers at the center of 3 long horizontal struts on the outer perimeter. Finally, 3 dampers are also at the center of each Down Rod to dampen the fundamental string mode of the rods. 

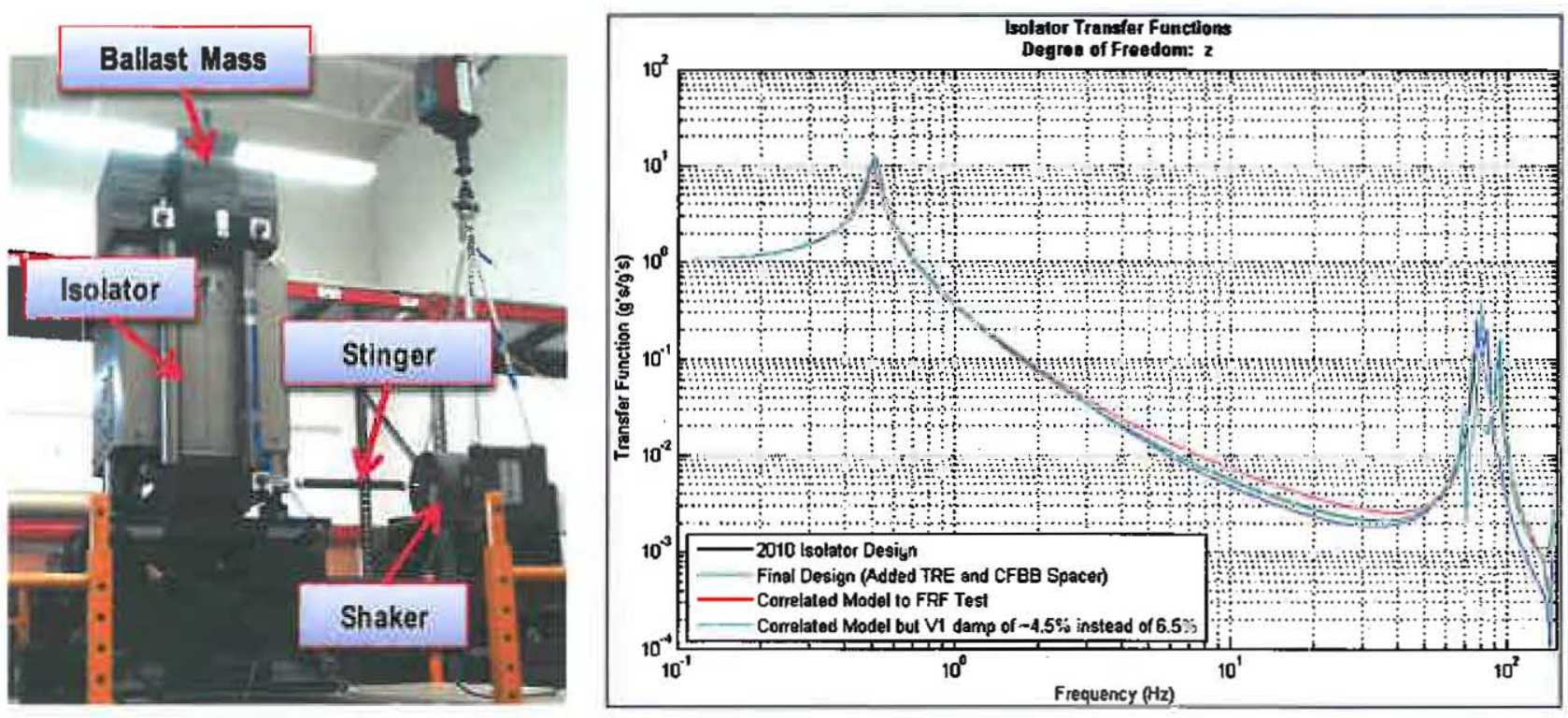

Figure 5 Vibration isolator under test and the transmissibility graph.

Table 1 FEM prediction results of vibration effects against optical test requirements.

\begin{tabular}{|l|l|l|l|}
\hline \multicolumn{2}{|c}{ Title Requirement } & Prediction \\
\hline PM Figure Error Vibration & $<4 \mathrm{~nm}$ RMS & $3.6 \mathrm{~nm}$ w/38 samples & $11 \%$ \\
\hline \multirow{2}{*}{ COCOA to PM } & $+/-25 \mu \mathrm{m}$ piston & $+/-0.2 \mu \mathrm{m}$ piston & $99 \%$ \\
& $+/-15 \mu \mathrm{m}$ decenter & $+/-12 \mu \mathrm{m}$ decenter & $18 \%$ \\
\hline Relative segment velocity & $+/-22.1 \mu \mathrm{m} /$ second & $+/-8 \mu \mathrm{m} /$ second & $65 \%$ \\
\hline
\end{tabular}

As a result, the predictions now meet the requirements for optical testing with the COCOA interferometer as shown in the table above (the sampling will be explained below). As mentioned before, the PM figure error results from the relative movement between the mirror segments. Lateral mis-alignment between the primary mirror (PM) and the COCOA causes mostly tilt fringes in the interferograms. Excessive fringe density increases the noise in the calculation of the wavefront and can lead to aliasing if the fringe density exceeds the Nyquist limit of the interferometer camera. The segment velocity is covered in the next section.

Figure 6 shows which vibration frequencies have a significant effect on wavefront error. 


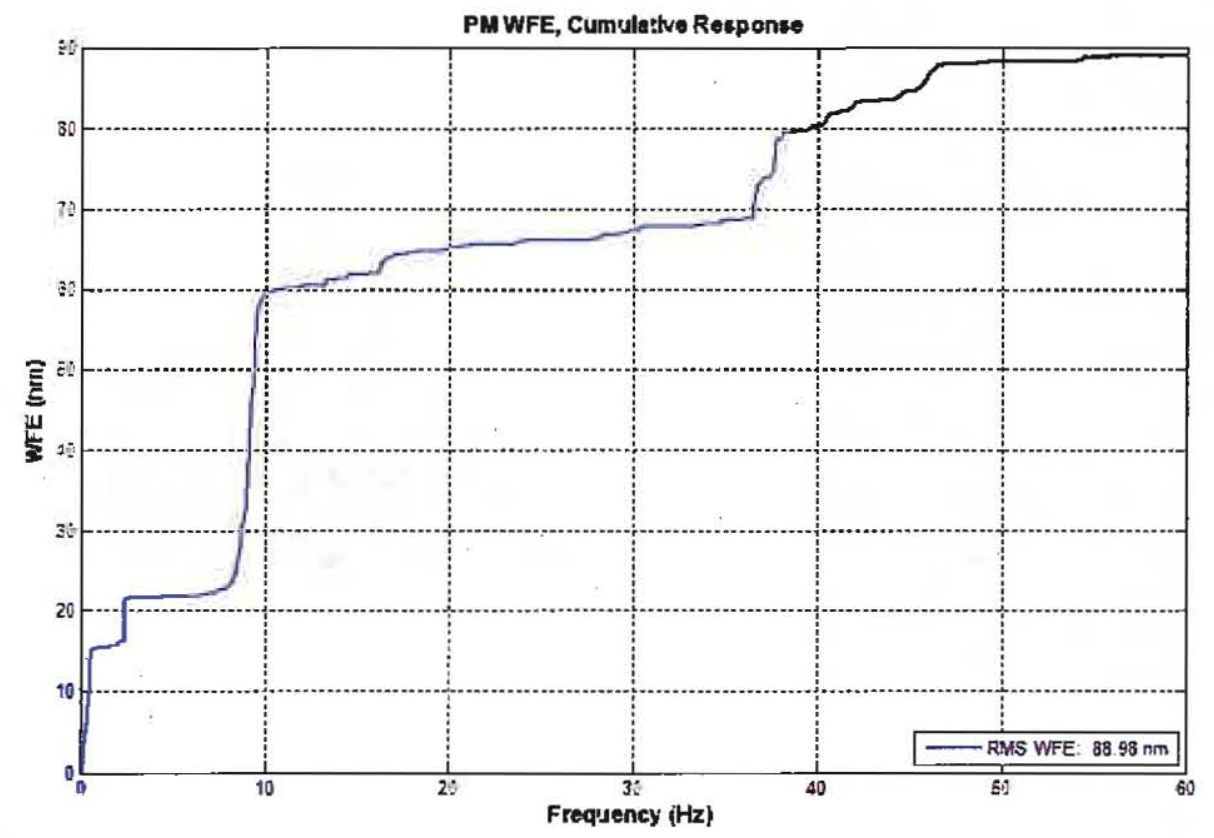

Figure 6 Sensitive vibration frequencies of PM wavefront error measurement.

\section{INTERFEROMETER OPERATION AND MEASUREMENT REDUCTION}

The interferometer is an instantaneous phase-shifting interferometer. In each exposure the beam propagates through waveplates and a micro polarizer pixel array mask that splits the beam into four different phases simultaneously onto the detector. This provides insensitivity to vibration for a single wavelength. However, the JWST optical test requires at least 2 wavelengths to decipher the phase separation between mirror segments in case the separation is greater than $1 / 4$ of a wavelength in distance. The interferometer cannot measure multiple wavelengths simultaneously. Step height movement between exposures leads to uncertainty in the phase separation. For the interferometer used in the JWST optical test, the duration between exposures varies from approximately 50 to 200 microseconds depending on wavelength and intensity. The maximum movement for the limiting wavelength-pair case is approximately 5 nanometers. The maximum velocity becomes the 22.1 micrometers per second stated in the table above.

The central limit theorem can be invoked in the measurement process. The interferometer captures multiple exposures at a $7 \mathrm{~Hz}$ rate. Averaging the exposures reduces the uncertainty of the vibration by a ratio of $1 / \mathrm{Vn}$ where $\mathrm{n}$ is the number of exposures. The FEM predicts surface figure variation due to vibration of 22 nanometers. With 38 exposures the uncertainty reduces to $(22 / \sqrt{38}=) 3.6 \mathrm{~nm}$. At $7 \mathrm{~Hz}, 38$ exposures equates to a period of 5.4 seconds. The number of exposures will actually be larger and over a longer time period to properly average slower frequency movement.

The interferometer successfully phased and tested the $1 / 6^{\text {th }}$ scale JWST testbed telescope built at Ball Aerospace Technologies. Figure 7 shows a picture of the telescope and a histogram of the displacement of one of the segments as measured by the interferometer. The phasing and figure error measured by the interferometer was favorably compared with phase retrieval wavefront sensing. 

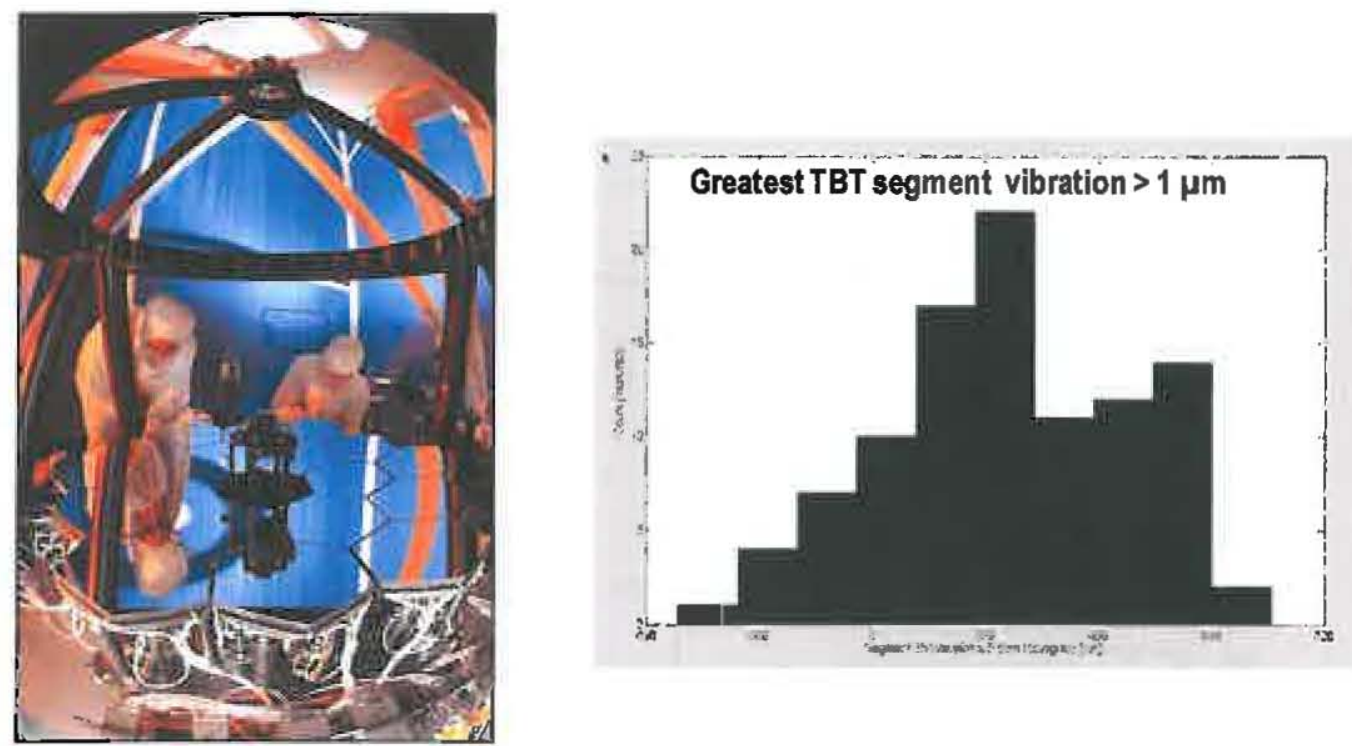

Figure 7 Picture of 1/6 scale telescope at Ball Aerospace Technologies and histogram of the segment with the largest vibration amplitude.

\section{SUMMARY .}

Although the JWST optical test facility was not originally built for optical testing, the optical measurement is achievable with the use of isolators, a test configuration designed to minimize vibration, strategic placement of dampers, and the use of a phase-shifting interferometer with the ability to average hundreds of interferograms to reduce the effect of vibration.

\section{ACKNOWLEDGEMENTS}

The optical test design and analysis was supported by the JWST contract NNG11FD64C with NASA GSFC. The JWST system is a collaborative effort involving NASA, ESA, CSA, the Astronomy community and numerous principal investigators. 